\title{
NANOSPONGE: A NOVEL NANO DRUG CARRIER
}

\author{
Anmol Eldose ${ }^{1 *}$, Parmar Twinkle ${ }^{2}$, Shah Honey ${ }^{3}$, Patel Jyoti ${ }^{4}$, Zala Twinkle ${ }^{5}$, Jain Hitesh ${ }^{6}$, Upadhyay Umesh ${ }^{7}$ \\ *1,2,3,4,5,6,7 Sigma Institute of Pharmacy, Vadodara, Gujarat, India, 390019 Email: anmoleldose38@ gmail.com \\ Mobile: +91-9624697442
}

*Corresponding Author: -

Email: anmoleldose38@gmail.com

\begin{abstract}
: -
Nanotechnology, a multi-disciplinary science has received considerable attention in the recent times in the discovery of new chemical entities, diagnosis and treatment of several diseases. Nanomedicine has developed many drug delivering systems like nanoparticles, nano-emulsions, nano-suspensions, nanosponges etc., to overcome the problems of bioavailability out of which nanosponge is an advanced drug delivery system which offers diverse advantages than the other available systems. Targeted drug delivery to specific sites is the significant problem which is being faced by the researchers. The development of new colloidal carrier called nanosponges has the potential to solve these problems. Nanosponge is a novel and emerging technology which offers controlled drug delivery for topical use. Nanosponges are solid, porous, biocompatible, and tiny in size with three-dimensional structure and nanomeric cavity size which have unique ability to entrap wide variety of drugs. Nanosponges can be used as a carrier for biocatalysts in the delivery and release of enzymes, proteins, vaccines and antibodies. Nanosponges are porous polymeric delivery systems that are small spherical particles with large porous surface. The nanosponges have the ability to include either lipophilic or hydrophilic drugs and release them in a controlled and predictable manner at the target site. By controlling the ratio of polymer to the cross-linker the particle size and release rate can be modulated.
\end{abstract}

Keywords: - Nanosponge, Quasi-emulsion solvent diffusion, Resilency, Solvent method, Ultra soundAssisted synthesis

\section{(ㄷ) (\$) (1)}




\section{INTRODUCTION}

The drug delivery technology has certainly a new interest for drugs by providing them new life through their therapeutic targets. Target oriented drug administration with improvements in therapeutic efficacy, reduction in side effects and optimized dosing regimen, shall be the leading trends in the area of therapeutics. Targeted drug delivery implies for selective and effective localization of pharmacologically active moiety at preidentified (preselected) target in therapeutic concentration, while restricting its access to non-target normal cellular linings and thus minimizing toxic effects and maximizing therapeutic index of the drug ${ }^{[1]}$. Nanosponges are a new class of materials and made of microscopic particles with few nanometres wide cavities, in which a large variety of substances can be encapsulated ${ }^{[2]}$. These particles are capable of carrying both lipophilic and hydrophilic substances and of improving the solubility of poorly water-soluble molecules ${ }^{[3]}$. These tiny sponges can circulate around the body until they encounter the specific target site and stick on the surface and begin to release the drug in a controlled and predictable manner. Because the drug can be released at the specific target site instead of circulating throughout the body it will be more effective for a particular given dosage ${ }^{[4]}$. The average diameter of a nanosponge is below $1 \mu \mathrm{m}$ as shown in Figure No. 1 but fractions below $500 \mathrm{~nm}$ can be selected, micro sponges are 10-25 microns in diameter. They can also decrease side effect and protect drug from degradation ${ }^{[5,6]}$.

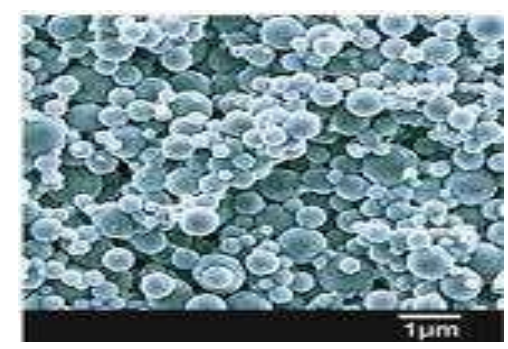

Fig.1: Porous Nanosponges

Nanosponges can encapsulate various types of molecules by forming inclusion and non-inclusion complexes [7]. Nanosponges in the presence of compounds possessing magnetic properties can be magneties. Their crystalline property maintains the loading capacity and it may be either paracrystalline or in crystalline form [8].

The nanosponges are solid in nature and can be formulated as oral, parenteral, topical or inhalational dosage forms. For oral administration, these may be dispersed in a matrix of excipients, diluents, lubricants and anticaking agents which is suitable for the preparation of tablets or capsules. For parenteral administration, these can be simply mixed with sterile water, saline or other aqueous solutions ${ }^{[9,10]}$.

\section{Advantages ${ }^{[11-14]}$ :}

1. This technology provide entrapment of active contents and side effects are less.

2. It provides improved stability, elegance and formulation flexibility.

3. It is non-mutagenic.

4. It provides extended-release condition which is continuous action up to $12 \mathrm{hr}$.

5. Drug is protected from degradation.

6. Therapeutic provide onset of action. Formulations are cost effective.

7. It can be used to mask unpleasant flavours and to convert liquid substances to solids.

8. The drug profiles can be varied from fast, medium to slow release in case of dosing therapy.

\section{Classification of Nanosponges ${ }^{[15,16]}$ \\ 1. Encapsulating nanoparticles}

This type is represented by nanosponges and nanocapsules. Nanosponges such as alginate nanosponge, which are sponge like nanoparticles containing many holes that carry the drug molecules in their aqueous core.

E.g. Nanosponges such as alginate nanosponge, which are sponge like nanoparticles containing many holes that carry the drug molecules. Nano capsules such as poly (iso-butyl-cyanoacrylate) are also encapsulating nanoparticles.

\section{Complexing nanoparticles}

This type of nanoparticles attracts the molecules by electrostatic charges.

\section{Conjugating nanoparticles}

This type of nanoparticles links to drugs through covalent bonds. As compared to the other nanoparticles, they are insoluble both in water and organic solvents, porous, non-toxic and stable at high temperature up to $300^{\circ} \mathrm{C}$. They are able to capture, transport and selectively release a huge variety of substances because of their 3D structure containing cavities of nanomeric size and tuneable polarity.

\section{Composition of nanosponges ${ }^{[17-20]}$}

Nanosponges are complex structures, normally built up from long linear molecules that are folded by cross linking into a more or less spherical structure, about the size of a protein. Typical nanosponges have been constructed from cyclodextrins cross-linked with organic carbonates. Nanosponges mainly consists three components. They are,

A. Polymer B. Cross linking agent C. Drug substance 


\section{Polymer}

Type of polymer used can influence the formation as well as the performance of Nanosponges. For complexation, the cavity size of nanosponge should be suitable to accommodate a drug molecule of particular size. The ability of the polymer to be cross-linked depends on the functional groups and active groups to be substituted. The selection of polymer depends on the required release and the drug to be enclosed. The polymers can be used to enclose the drug or to interact with the drug substance. For the targeted drug release the polymer should have the property to attach with the specific ligands.

\section{Crosslinking agent}

Selection of crosslinking agent depends on the structure of polymer and the drug to be formulated. The list of polymers and crosslinking agents used for the synthesis of nanosponges are presented in Table-1.

Drug substance

Drug molecules to be formulated as nanosponges should have certain characteristics mentioned below. a. Molecular weight between 100 and 400 .

b. Drug molecule consists of less than five condensed rings.

c. Solubility in water is less than $10 \mathrm{mg} / \mathrm{mL}$

d. Melting point of the substance is below $250^{\circ} \mathrm{C}$.

\section{Methods of preparation of nanosponges ${ }^{[21-22]}$}

1) Emulsion solvent diffusion method

In this method the two phases used are organic and aqueous. Aqueous phase consists of polyvinyl alcohol and organic phase include drug and polymer. After dissolving drug and polymer to suitable organic solvent, this phase is added slowly to the aqueous phase and stirred for two or more hours and then nanosponges are collected by filtration washed and then dried in air at room temp or in vacuum oven $40^{\circ} \mathrm{c}$ for $24 \mathrm{hrs}$.

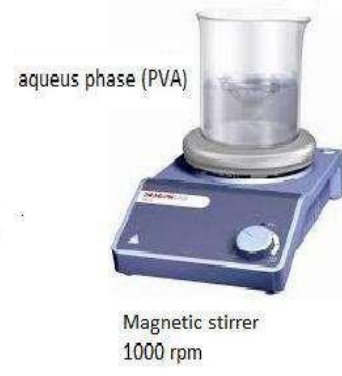

$1000 \mathrm{rpm}$

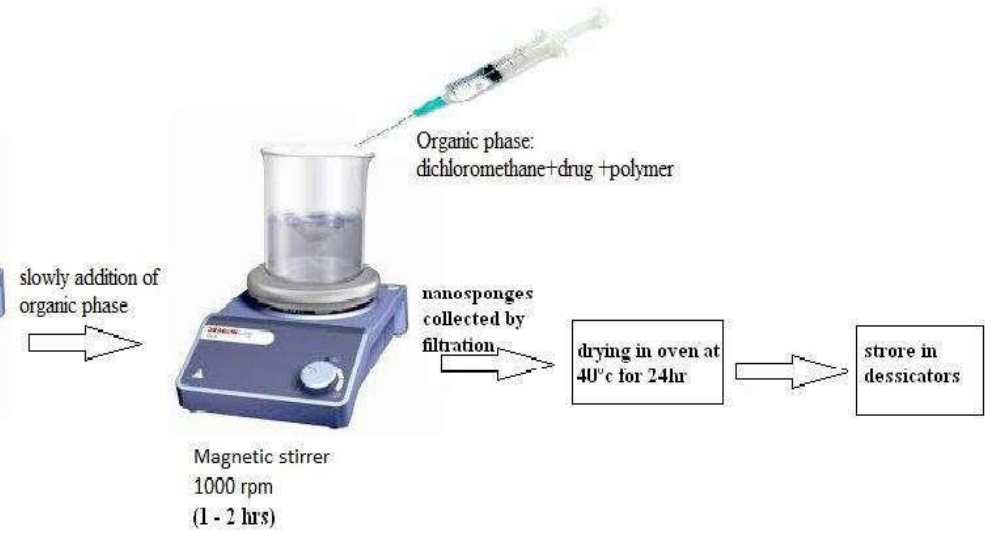

Fig.2. Preparation of nanosponges.

\section{2) Quasi-emulsion solvent diffusion}

This process involved formation of quasi-emulsion of two different phases"e i.e. internal phase and external phase similar to emulsions. The internal phase of drug--polymer solution made in a volatile solvent like ethanol or acetone or dichloromethane was added to external phase comprising the aqueous polyvinyl alcohol (PVA) solution with vigorous stirring. Triethylcitrate (TEC), which was added at an adequate amount in order to facilitate plasticity. Stirring lead to the formation of discrete emulsion globules called quasi-emulsion globules. Solvent was then extracted out from these globules to form insoluble, rigid microparticles

Following 60min of stirring, the mixture is filtered to separate the nanosponges. The nanosponges are dried in an airheated oven at $40^{\circ} \mathrm{c}$ for $12 \mathrm{hrs}$.

\section{3) Solvent method}

Mix the polymer with a suitable solvent, in particular in a polar aprotic solvent such as di methyl formamide, di methyl sulfoxide. Then add this mixture to excess quantity of the cross- linker, preferably in cross linker/polymer molar ratio of 4 to 16. The reaction was carried out at temperature ranging from $10^{\circ} \mathrm{C}$ to the reflux temperature of the solvent, for time ranging from 1 to $48 \mathrm{~h}$. After completion of the reaction, allow the solution to cool at room temperature, then add the product to large excess of bi distilled water and recover the product by filtration under vacuum and subsequently purify by prolonged soxhlet extraction with ethanol. Dry the product under vacuum and grind in a mechanical mill to obtain homogeneous powder.

\section{4) Ultrasound-Assisted synthesis}

In this method nanosponges can be obtained by reacting polymers with cross-linkers in the absence of solvent and under sonication. The nanosponges obtained by this method will be spherical and uniform in size. Mix the polymer and the cross-linker in a particular molar ratio in a flask. Place the flask in an ultrasound bath filled with water and heated to $90^{\circ} \mathrm{C}$. Sonicate the mixture for 5hours. Then allow the mixture to cool and break the product roughly. Wash the product 
with water to remove the non-reacted polymer and subsequently purify by prolonged soxhlet extraction with ethanol. Dry the obtained product under vacuum and store at $25^{\circ} \mathrm{C}$ until further use.

\section{Evaluation of nanosponges ${ }^{[23-27]}$ :}

1) Particle size determination:

Particle size analysis of loaded and unloaded nanosponges performed by laser light diffractometry or Malvern zeta sizer. Cumulative graph is maintained or plotted as particle size against time to study effect of particle size on drug release. Particles size greater then 30m impart gritty feeling and particles of sizes between 10 and $25 \mathrm{~m}$ preferred and used in final optimised formulation.

\section{2) Morphology and surface topography:}

For preparation of nanosponges in terms of morphology they are coated with gold-palladium under an atmosphere of orgon at room temperature and surface structure studied by scanning electron microscopy.

\section{3) Determination of loading efficiency and production yield:}

The loading efficiency $(\%)$ of nanosponges calculated according to the equation.

$$
\text { Drug loading efficiency }=\left(\begin{array}{c}
\begin{array}{c}
\text { Practical amount of } \\
\text { drug loaded } \\
\text { Theoretical amount of } \\
\text { drug loaded }
\end{array}
\end{array}\right) \times 100
$$

The yield of nanoparticles can be determined by calculating initial weight of nanosponges as,

$$
\text { Percent yield }=\frac{\text { Actual yield }}{\text { Theoretical yield }} \times 100 \%
$$

\section{6) Dissolution tests:}

Dissolution profile of nanosponges are studied using dissolution apparatus USP having a modified basket consist of $5 \mathrm{ml}$ stainless steel mesh with a speed of rotation around $150 \mathrm{rpm}$. Proper dissolution medium is selected and solubility of active contents are considered to ensure sink conditions. Proper analytical method are used for the sample form dissolution medium.

\section{Characterization of nanosponges ${ }^{[28-32]}$ : \\ 1) Thermo-analytical methods:}

It shows the changes occur in drug substance before undergoing thermal degradation of nanosponges. The change of drug may be melting, evaporation, oxidation, decomposition or polymeric transition. Changes in drug substance indicates formation of complex. DTA and DSC observed for broadening, shifting and appearance of new peaks. If changes in weight loss occurs can provide evidence for formation of inclusion complexes.

\section{2) Microscopy studies:}

Scanning electron microscopy and Transmission electron microscopy used to study microscopic aspects of drug nanosponges and product. Difference in crystallization state of raw materials and product seen under electron microscope.

\section{3) Solubility studies:}

It is the most widely used approach to study inclusion complex and mainly described by Higuchi and Connor's equation for phase solubility and helps in examine the effect on solubility of drug by nanosponge.

\section{4) X-ray diffractometry:}

Powder x-ray diffractometry used to detect inclusion complex in solid state. If we consider liquid then it has no diffraction pattern of their own and totally differs from in complexed nanosponge. If drug is a solid substance comparison should be made between diffractogram of assumed complex and mechanical mixture of dry and it alters diffraction patterns. A diffraction pattern of a physical mixture results from combination of two components. But complexes having diffraction pattern mainly differs from the constituent they contain and give rise to "new" solid phase having different diffractograms. They give rise to different peaks for a mixture and useful in determining chemical decomposition and complex formation. -Single crystal Xray structure analysis: It may also be used to determine the inclusion structure and way it interact. Interaction between host and external molecules can be determined and a precise relationship can be established.

\section{6) Loading efficiency:}

It describes the efficiency or determined by quantitative estimation of drug loaded into nanosponges by UV spectrophotometer \& HPLC methods.

\section{7) Zeta potential:}

It measures surface charge and by adding an electrode it can be measured in particle size equipment.

Applications of Nanosponges ${ }^{[33-38]}$ :

- Nanosponges as chemical sensors

- Nanosponge for oral delivery 
- Solubility enhancement

- Nanosponges as a carrier for biocatalysts and release of enzymes, proteins, vaccines and antibodies

- Antiviral application

- In Cancer

- In the removal of organic matter to produce ultrapure water for power regeneration.

- Nanosponges as a sustained delivery system

- Nanosponges for protein delivery

- Nanosponges in enzyme immobilization

- As a carrier for delivery of gases

- As a protective agent from light and degradation

\section{Conclusion}

The nanosponge systems have been found to have good potential for prolonged drug release and therefore can be beneficial for use in the treatment of various chronic diseases. Additional benefits such as dose reduction, reduced frequency of administration and avoiding related systemic side effects can be produced. Due to their small particle size and spherical shape these can be developed as different dosage forms like oral, parenteral and topical preparations. Nanosponge technology offers entrapment of ingredients and thus reduced side effects, improved stability, increases elegance and enhanced formulation flexibility. Besides their application in the drug delivery field, potential applications exist for cosmetics, biomedicine, bioremediation processes, agro chemistry, and catalysis, among others.

Kriajevska M.V., M.N. Cardenas, M.S. Grigorian , N.S. Ambartsumian, G.P. Georgiev, E.M. Lukanidin (1994) Nonmuscle myosin heavy chain as a possible target for protein encoded by metastasis-related mts-1 gene. J Biol Chem, 269(31):19679-19682

\section{References}

[1].Vyas S, Khar RK (2008) Targeted and Controlled Drug Delivery- Novel Carrier Systems. Molecular Basis of Targeted Drug Delivery. CBS Publishers and Distributors. New Delhi, 38- 40.

[2].Trotta F, Cavalli R, Tumiatti W, Zerbinati O,Rogero C, Vallero R (2007) Ultrasound- Assisted synthesis of Cyclodextrin-based nanosponges. EP1, 786: 841.

[3].David F. Nanosponge drug delivery system more effective than direct injection. www.physorg.com

[4].01.06.2010, accessed on 20.12.2011.

[5].Jenny A, Merima P, Alberto F, Francesco T (2011) Role of $\beta$-cyclodextrin nanosponges in propylene photo oxidation. Carbohydrate Polymers, 86:127-135.

[6].Cavalli R, Trotta F and Tumiatti W (2006) Cyclodextrin-based Nanosponges for Drug Delivery. Journal of Inclusion Phenomena and Macro Chemistry, 56(1-2):209-213.

[7].Nacht S, Kantz M (1992) The Microsponge: A Novel Topical Programmable Delivery System: Topical Drug Delivery Systems. Edited by David W. O. and Anfon H. A., 42: 299-325.

[8].E.K Patel, RJ Oswal (2012) Nanosponge and Microsponges: A Novel Drug Delivery System International Journal of Research in Pharmacy and Chemistry, 2(2): 237-244.

[9].Lala R, Thorat A, Gargote C (2011). Current trends in $\beta$-cyclodextrin based drug delivery systems .Int J Res Ayur Pharm, 2(5): 1520-1526.

[10]. Leslie Z, Benet (2007) BCS and BDDCS. Bioavailability and Bioequivalence: Docus on Physiological Factors and Variability. Department of pharmaceutical sciences, University of California, San Francisco, USA.

[11]. Renuka S, Roderick B, Kamla P (2011) Evaluation of Kinetics and Mechanism of Drug Release from Econazole nitrate Nanosponge Loaded Carbapol Hydrogel. Ind J Pham Edu Res, 45(1):25-31.

[12]. NileshJ, Ruchi J, Navneet T, Brham PG, Deepak KJ (2010) Nanotechnology: A Safe and Effective Drug Delivery Systems. Asian Journal of Pharmaceutical and Clinical Research, 3(3), 159-165.

[13]. Nacht S, Kantz M (1992) The microsponge: a novel topical programmable delivery system, In: Topical Drug Delivery Systems. David W.O and Anfon H.A (ED), 42.

[14]. Delattre L, Delneuville I (1995) Biopharmaceutical aspects of the formulation of dermatological vehicles. J Eur Acad Derm Vener, 5:70.

[15]. http://Sciencematters, Unimelb.edu.au/ 2011/05/nanosponges for targeted- cancer-treatment/visited on 12/10/2011.

[16]. Sharma R, Roderick B, and Pathak K (2011) Evaluation of kinetics and mechanism of drugrelease from Econazole nitrate Nanosponge loaded carbopol Hydrogel. Indian J of Pharma Edu and research, 45(1):25-31.

[17]. Cavalli R, Trotta F, Tumiatti V (2006) Cyclodextrin-based nanosponges for drug delivery. J Incl Phenom Macrocyl Chem , 56: 209- 213

[18]. Selvamuthukumar Subramanian et al (2012) Nanosponges: A Novel Class of Drug Delivery System - Review. J Pharm Pharma Sci, 15(1): 103 - 111.

[19]. Geeta Yadav (2013) Nanosponges: A boon to the targeted drug delivery system (2013) Journal of Drug Delivery \& Therapeutics, 3(4): 151-155.

[20]. Uday B., Manvi FV, et.al (2013) Recent Advances in Nanosponges as Drug Delivery System. International Journal of Pharmaceutical Sciences and Nanotechnology, 6(1):1934-1944.

[21]. Jilsha G, Vidya Viswanad (2013) Nanosponges: A Novel Approach of Drug Delivery System. Int. J. Pharm. Sci. Rev. Res, 19(2): 119-123. 
[22]. Embil K., and Nacht S.(1996) The microsponge delivery system atopical delivery system with reduced irritancy incorporating multiple triggering mechanisms for the releaseof actives. J Microencapsule, 13: 575-88.

[23]. Mishra M.K., Shikhri M., Sharma R., and Goojar M.P. (2011) Optimization, formulation, development and characterization of Eudragit RS 100 loaded microsponges and subsequent colonic delivery. Int J of Drug Discovery And herbal Research, 1(1): 8-13.

[24]. Martin A., Swarbrick J., and Cammarrata A. (2003) In: Physical Pharmacy-Physical Chemical Principles in Pharmaceutical Sciences, 3: 527.

[25]. Emanuele A., and Dinarvand R. (1995), Preparation, Characterization and Drug Release from Thermoresponsive Microspheres. Int JPharm, 237:42.

[26]. Kilicarslan M., and Baykara T. (2003) The effect of the drug/polymer ratio the properties of Verapamil $\mathrm{HCl}$ loaded microspheres. Int JPharm, 252:99-109.

[27]. Barkai A., Pathak V., and BenitaS (1990) Polyacrylate (Eudragit retard) microspheres for oral controlled release of nifedipine, Formulation design and process optimization. Drug Dev Ind Pharm, 16:2057-2075.

[28]. Wester R.,Patel R.,Natch S.,Leyden J.,Melendres J.,and Maibach H. (1991) Controlled release of benzoyl peroxide from a porous microsphere polymeric system can reduce topical irritancy, J. Am. Acad. Derm, 24:720-726.

[29]. Ramnik S., Nitin B., Jyotsana M., Horemat S. (2010) Characterization of Cyclodextrin Inclusion complexes $-A$ Review. J Pharm Sci Tech, 2(3):171-183.

[30]. Maravajhala V., Papishetty S., Bandlapalli S. (2012) Nanotechnology in the development of drug delivery system, International journal of pharmaceutical sciences \& research, 3(1): 84-96.

[31]. Rao M. R., Bajaj A. N., Pardeshi A. A., Aghav S. S. (2012) Investigation of Nanoporous colloidal carrier for solubility enhancement of Cefpodoxime proxetil, Journal of pharmacy research, 5(5): 2496-2499.

[32]. Ajay V, Preetam N, et,al (2014) Nanosponges: A Benefication For Novel Drug Delivery, International Journal of Pharm. Tech Research, 6(1):11-20.

[33]. Flowerlet Mathew, et.al (2014) A Review on Targeted Drug Delivery through Nanosponge, International Journal of Universal Pharmacy and Bio Sciences 3(4):227-47

[34]. Zuruzi S., MacDonald N.C., Moskovits M., and Kolmakov A. (2007) Metal oxide "nanosponges" as chemical sensors: Highly sensitive detection of hydrogen using nanosponge titania; Angewandte Chemie, International Edition, 46 (23): 4298-4301.

[35]. Swaminathan S., Vavia P.R., Trotta F. (2007) Formulation of beta cyclodextrins based nanosponges of itraconazole. J Incl Phenom Macro Chem., 57:89-94.

[36]. Gilardi G., Trota F., Cavalli R., Ferruti P., Ranucc iE., Di Nardo G., Roggero C., Tumiatti V. (2009) Cyclodextrin nanosponges as carrier forbiocatalysts, and in the delivery and release of enzymes, proteins, vaccines and antibodies, WO2009149883 A1.

[37]. Wong V.N., Fernando G., Wagner A.R., Zhang J,Kinsel G.R., Zauscher S., Dyer D.J. (2009) Separation of peptides with polyionic nanosponges for MALDIM Sanalysis. Langmuir,25(3):145965.

[38]. Ansari K.A., Torne S., Vavia P.R., Trotta F., Cavalli R. (2011) Cyclodextrin - Based Nanosponges for Delivery of Resveratrol: In Vitro Characterization, Stability, Cytotoxicity and Permeation Study, AAPS Pharm Sci Tech,12(1): 279-86.

[39]. Rosalba M, Roberta C, Roberto F, Chiara D, Piergiorgio P, Leigh E, Li S, Roberto P (2011). Antitumor activity of nanosponge-encapsulated Camptotechin in human prostate tumors. Cancer Res, 71:4431. 